\title{
Gas Diffusion in Coal Powders is a Multi-rate Process
}

\author{
Simon A. Mathias ${ }^{1} \cdot$ Marco Dentz $^{2} \cdot$ Qingquan Liu $^{3}$
}

Received: 18 July 2019 / Accepted: 2 December 2019 / Published online: 10 December 2019

(c) The Author(s) 2019

\begin{abstract}
Gas migration in coal is strongly controlled by surface diffusion of adsorbed gas within the coal matrix. Surface diffusion coefficients are obtained by inverse modelling of transient gas desorption data from powdered coals. The diffusion coefficient is frequently considered to be dependent on time and initial pressure. In this article, it is shown that the pressure dependence can be eliminated by performing a joint inversion of both the diffusion coefficient and adsorption isotherm. A study of the log-log slope of desorbed gas production rate against time reveals that diffusion within the individual coal particles is a multi-rate process. The application of a power-law probability density function of diffusion rates enables the determination of a single gas diffusion coefficient that is constant in both time and initial pressure.
\end{abstract}

Keywords Coal-bed methane $\cdot$ Diffusion coefficient $\cdot$ Gas desorption $\cdot$ Multi-rate

\section{Introduction}

There is much interest in measurement of gas diffusion coefficients for coal. Such coefficients are required for field-scale coal-bed-methane (CBM) simulators to plan and forecast the performance of CBM production operations. Coal beds generally exhibit an orthogonal set of fractures. Fractures in coal are referred to as cleats. The surrounding blocks of coal are typically referred to as the matrix. Methane gas is adsorbent in coal. Gas adsorption is a pressure-dependent process with adsorption increasing with increasing pressure. $\mathrm{CBM}$ production involves reducing pressure in the coal bed by fluid production (this can be

Simon A. Mathias

s.a.mathias@durham.ac.uk

Marco Dentz

marco.dentz@gmail.com

Qingquan Liu

cumtsafe@cumt.edu.cn

1 Department of Engineering, Durham University, Durham, UK

2 Spanish National Research Council (IDAEA-CSIC), 08034 Barcelona, Spain

3 Key Laboratory of Coal Methane and Fire Control, Ministry of Education, China University of Mining and Technology, Xuzhou, China 
water and/or gas). The reduced pressure causes gas to desorb and migrate through the cleat system.

Due to very small permeability, migration of gas within the coal matrix is dominated by diffusion. Three modes of gas diffusion are generally considered (Zhao et al. 2019): (1) free diffusion of desorbed gas in the pore-space of the coal matrix; (2) Knudsen diffusion of desorbed gas in the pore-space of the coal matrix; (3) surface diffusion of adsorbed gas within layers of adsorbed gas in the coal matrix.

Free diffusion of desorbed gas within the coal matrix is not thought to be significant because the gas molecules are a similar size to the pore-sizes in the coal matrix of concern. Instead, Knudsen diffusion is likely to dominate, whereby diffusion is enhanced by the bouncing of gas molecules on the side of the pores. Nevertheless, many simulators assume only surface diffusion of adsorbed gas occurs within the matrix with all gas desorption taking place at the cleat face (King et al. 1986; Ye et al. 2014; Zang and Wang 2016; Miao et al. 2018).

A common method of measuring gas diffusion coefficients is the so-called "particle method" (Dong et al. 2017). This involves grinding coal into a powder and sieving out particles within a fixed diameter interval. These are then packed into a reactor vessel. Methane gas is injected into the vessel until a designated pressure is reached. The vessel is then exposed to atmospheric pressure, and the volume of gas produced from the vessel is recorded with time. The diffusion coefficient of the coal is estimated by calibrating a mathematical model of gas diffusion to the observed gas production time-series (Guo et al. 2016; Yue et al. 2017; Dong et al. 2017; Cheng-Wu et al. 2018).

The observed diffusion process is generally thought to represent the surface diffusion of adsorbed methane within the ground coal particles. A problem frequently encountered is that a mathematical model of Fickian diffusion in a homogeneous spherical particle is unable to simulate both the early and late time portions of the experiment. Instead, a weighted mean of responses from two spherical diffusion models with different diffusion coefficients is often applied, generally referred to as a bidisperse model (Smith and Williams 1984; Clarkson and Bustin 1999; Wang et al. 2014). Whilst such a model has sufficient degrees of freedom (two diffusion times and a weighting coefficient) to fit the observed data of concern, the physical basis of the conceptual model is weak. The model represents a mixture of particles with two different sizes and/or two different diffusion coefficients. Whilst it is conceivable that there should be a continuum of different particle sizes present in such experiments, it is unclear why the distribution should be dominated by two specific sizes in particular.

More recently, spherical diffusion models with transient diffusion coefficients have been adopted, whereby a spatially uniform diffusion coefficient is defined using a heuristic function that continually declines with time (Dong et al. 2017; Yue et al. 2017; Zhao et al. 2017; Cheng-Wu et al. 2018). Such an approach leads to simple to evaluate analytical solutions, which are straightforward to accurately calibrate against observed data. However, there is no physical basis to justify allowing a spatially uniform diffusion coefficient to decline with time in this context.

Jiang et al. (2013), Kang et al. (2015, 2016) and Fan et al. (2016) alternatively proposed to use time and space-fractional diffusion equations in order to describe gas production curves over the full time range. This more phenomenological approach is driven by the general use of fractional diffusion models for anomalous diffusion processes and does not require transient diffusion coefficients. However, whilst the use of fractional space derivatives is motivated by a possibly fractal grain structure within the coal particles, a physical basis for using time-fractional derivatives is unclear in this context. Furthermore, 
a time-fractional approach predicts a persistent power-law decay in production rate, which is generally not the case (Dentz et al. 2004; Meerschaert et al. 2008). Instead, production rates tend to exponentially cut off at a characteristic time-scale, as will be shown using experimental gas desorption data later in the article.

The fact that a transient diffusion coefficient or a time-fractional derivative is required implies that there is missing physics within the conventional Fickian diffusion model. Previous researchers have suggested that the missing physics of concern includes free and Knudsen diffusion within the coal matrix (Dong et al. 2017; Liu et al. 2018; Liu and Lin 2019).

Wang et al. (2017) found that the need for a transient diffusion coefficient can be eliminated by using a dual-porosity model whereby coal particles are assumed to comprise a micro- and macro-pore-space. A dual-porosity framework will give rise to at least two additional fitting parameters as compared to a single porosity diffusion model and will therefore be much better at matching observed experimental data (Zang et al. 2019). Of note is that the gas production data from "particle-method" experiments are presented as desorbed gas volume as a function of time. However, a better diagnostic approach, not typically used in the literature, is to study desorbed gas production rate as a function of time on $\log -\log$ axes. Dual-porosity phenomena will manifest itself as two connected offset straight lines both with $\log -\log$ slopes of -0.5 , one for the macro-pores and another for the micropores. This will similarly be the case for the so-called bidisperse model.

A - 0.5 slope is characteristic of spherical or near spherical particles of the same diameter and homogeneous spatial structure. A single straight line with a log-log slope, which is not equal to -0.5 is indicative of multi-rate phenomena (MRP), whereby there are multiple diffusion rates simultaneously present (Haggerty et al. 2000, 2001; Gouze et al. 2008). A distribution of particle sizes and shapes as well as heterogeneous spatial structure will give rise to such phenomena. 3D laser scanning of ground coal particles, such as those used in the aforementioned "particle method" experiments, reveals that individual coal particles are frequently aspherical and angular (Koekemoer and Luckos 2015). Such particles appear to be assembled from conglomerations of smaller particles comprising a wide range of possible sizes, which would provide a good physical basis for MRP in this context. Accommodating for MRP will likely remove the need for adopting a transient diffusion coefficient to simulate observed phenomena in experimental gas production data.

It is also noted that previous research has treated diffusion coefficient to be a function of the initial pressure of the experiment. However, the gas pressure within the packed bed pore-space is assumed to be at atmospheric pressure from the start of the experiment. The fact that different diffusion coefficients are required for different initial pressures points towards potential errors in the gas adsorption isotherm being adopted (which is pressuredependent). A frequently used adsorption isotherm is the Langmuir isotherm, which has two physical parameters. These parameters are generally obtained by calibrating the isotherm to an additional set of steady state desorption experiments (Guo et al. 2016; Yue et al. 2017; Dong et al. 2017).

Liu et al. (2017) were able to describe diffusion controlled, time-dependent swelling of coal matrix upon $\mathrm{CH}_{4}$ adsorption with a single diffusion coefficient that is constant with both pressure and time. It follows that it should be possible to describe gas production rates from gas desorption experiments with a constant diffusion coefficient as well.

The objective of this article is to demonstrate that gas production rates from gas desorption experiments using ground coal particles can be described using a single static diffusion coefficient that is independent of initial pressure when MRP are accounted for and the associated gas adsorption isotherm is obtained through a joint inversion of gas desorption data for all 
initial pressures studied. The demonstration is developed using experimental data previously presented by Dong et al. (2017).

The outline of this article is as follows: The experimental procedure of Dong et al. (2017) is introduced and described. A simple spherical diffusion model is presented. A new multi-rate model is developed assuming that diffusion rate is a stochastic process characterized by a truncated power-law probability density function, hereafter referred to as a stochastic power-law model. A joint inversion procedure is described involving simultaneous calibration of models to observed gas desorption data for multiple experimental initial pressures. A joint inversion of diffusion coefficient and adsorption isotherm is applied using the simple spherical diffusion model. The exercise is then repeated using the stochastic power-law model.

\section{Methods and Data}

\subsection{Description of Gas Desorption Experiments}

In this article, "particle method" experimental data, previously generated by Dong et al. (2017), are revisited. The experiments focus on anthracite from the Daning coal mine in the Qinshui Basin of China. The coal was ground into powder, and two samples were acquired, one with particles ranging from 1.0 to $3.0 \mathrm{~mm}$ in diameter (sample 1) and another with particles ranging from 0.5 to $1.0 \mathrm{~mm}$ in diameter (sample 2). The density of the coal, $\rho_{\mathrm{c}}$ $\left[\mathrm{ML}^{-3}\right]$, was measured as being $1.50 \mathrm{~g} \mathrm{~cm}^{-3}$.

For each experiment, a reactor vessel, of interior volume, $V_{\mathrm{v}}\left[\mathrm{L}^{3}\right]$, of $102 \mathrm{ml}$, was packed with a mass of coal powder, $M_{\mathrm{c}}[\mathrm{M}]$, of $50 \mathrm{~g}$. The vessel was then exposed to a vacuum for $24 \mathrm{~h}$ to remove all free and adsorbed gas. Following from this, the vessel was heated and maintained at a constant temperature, $T[\Theta]$, of $303.15 \mathrm{~K}$. Pure methane was subsequently injected into the vessel until a desired gas pressure, $P_{\mathrm{I}}\left[\mathrm{ML}^{-1} \mathrm{~T}^{-2}\right]$, was achieved. The vessel was maintained at $P=P_{\mathrm{I}}$ for $6 \mathrm{~h}$ to ensure gas adsorption equilibrium within the coal particles was achieved. The initial pressures studied were $0.25,0.5,1,2,3$ and $4 \mathrm{MPa}$.

The outlet of the reactor vessel was reduced to atmospheric pressure, $P_{0}\left[\mathrm{ML}^{-1} \mathrm{~T}^{-2}\right]$, $0.101 \mathrm{MPa}$, by linking the outlet to a closed gas sample bag, exposed externally to atmospheric conditions. Atmospheric pressure was reached at the vessel outlet after around $5 \mathrm{~s}$, and then, the vessel was connected to a gas measuring cylinder. The volume of gas entering the measuring cylinder was recorded at different times for a total of $120 \mathrm{~min}$.

The results of the experiment are presented as desorbed gas volume, $v_{\mathrm{d}}\left[\mathrm{L}^{3} \mathrm{M}^{-1}\right]$, in $\mathrm{ml}$ $\mathrm{g}^{-1}$. This represents the volume of gas at standard conditions (taken to be $303.15 \mathrm{~K}$ and $0.101 \mathrm{MPa}$ ), in $\mathrm{ml}$, desorbed from a gram of coal powder, after a given time, $t[\mathrm{~T}]$, since the reactor vessel was connected to the closed gas sample bag. This $v_{\mathrm{d}}$ term is calculated from (Guo et al. 2016)

$$
v_{\mathrm{d}} \equiv \frac{M_{\mathrm{d}}}{\rho_{\mathrm{g} 0} M_{\mathrm{c}}}=\frac{M_{\mathrm{gt}}-M_{\mathrm{gf}}}{\rho_{\mathrm{g} 0} M_{\mathrm{c}}}
$$

where $M_{\mathrm{d}}[\mathrm{M}]$ is the mass of desorbed gas, $M_{\mathrm{gt}}[\mathrm{M}]$ is the total mass of gas contained within both the closed gas sample bag and the measuring cylinder, $M_{\mathrm{gf}}[\mathrm{M}]$ is the total mass of free gas that will ultimately be released from the reactor vessel that is not thought to have previously been adsorbed to the coal and $\rho_{\mathrm{g} 0}\left[\mathrm{ML}^{-3}\right]$ is the density of gaseous methane at standard conditions. $M_{\mathrm{gf}}$ term is calculated from (Guo et al. 2016) 


$$
M_{\mathrm{gf}}=\left(V_{\mathrm{v}}-\frac{M_{\mathrm{c}}}{\rho_{\mathrm{c}}}\right)\left(\rho_{\mathrm{g} I}-\rho_{\mathrm{g} 0}\right)
$$

where $\rho_{\mathrm{g} I}\left[\mathrm{ML}^{-3}\right]$ is the density of gaseous methane at $P=P_{\mathrm{I}}$.

\subsection{Mathematical Models}

In this section, a simple spherical diffusion model is presented. We then develop a new multi-rate, stochastic power-law model by assuming that diffusion rate is characterized by a truncated power-law probability density function (PDF). When using the simple spherical diffusion model, the log-log slope of gas production rate as a function of time is restricted to -0.5 . Log- $\log$ slopes that deviate from -0.5 are indicative of multi-rate phenomena. An important advantage of the stochastic power-law model is that this log-log slope is explicitly controlled by an empirical parameter, $k$.

\subsubsection{Simple Spherical Diffusion Model}

For coal particles that are homogeneous spheres of equal size, $v_{\mathrm{d}}$ can be determined using the simple spherical diffusion model (Crank 1979; Dong et al. 2017)

$$
F \equiv \frac{v_{\mathrm{d}}}{v_{\mathrm{d} 0}}=1-\frac{6}{\pi^{2}} \sum_{n=1}^{\infty} \frac{1}{n^{2}} \exp \left(-\alpha \tau_{n}\right)
$$

where

$$
\begin{gathered}
v_{\mathrm{d} 0}=v_{\mathrm{L}}\left(\frac{P_{I}}{P_{\mathrm{L}}+P_{\mathrm{I}}}-\frac{P_{0}}{P_{\mathrm{L}}+P_{0}}\right) \\
\alpha=\frac{4 D_{\mathrm{A}}}{a^{2}} \\
\tau_{n}=\pi^{2} n^{2} t
\end{gathered}
$$

and $v_{\mathrm{L}}\left[\mathrm{L}^{3} \mathrm{M}^{-1}\right]$ and $P_{\mathrm{L}}\left[\mathrm{ML}^{-1} \mathrm{~T}^{-2}\right]$ are Langmuir isotherm parameters describing gas adsorption within the coal particles, $D_{\mathrm{A}}\left[\mathrm{L}^{2} \mathrm{~T}^{-1}\right]$ is the apparent diffusion coefficient, and $a[\mathrm{~L}]$ is the diameter of the coal particles. By fitting a Langmuir isotherm to steady state gas desorption data, Dong et al. (2017) previously found that $v_{\mathrm{L}}=36 \mathrm{ml} \mathrm{g}^{-1}$ and $P_{\mathrm{L}}=1.13$ MPa for the anthracite of concern.

Also of relevance is that the desorbed gas production rate, $\mathrm{d} v_{\mathrm{d}} / \mathrm{d} t$, is found from

$$
\frac{\mathrm{d} F}{\mathrm{~d} t} \equiv \frac{1}{v_{\mathrm{d} 0}} \frac{\mathrm{d} v_{\mathrm{d}}}{\mathrm{d} t}=6 \alpha \sum_{n=1}^{\infty} \exp \left(-\alpha \tau_{n}\right)
$$

The $v_{\mathrm{d} 0}$ parameter represents the maximum volume of gas per unit mass of coal that can be desorbed from the experiment. The $\alpha$ parameter represents the characteristic diffusion rate of the spherical particle under consideration. 


\subsubsection{Stochastic Power-Law Model}

If the diffusion rate parameter, $\alpha\left[\mathrm{T}^{-1}\right]$, is stochastic and characterized by a $\mathrm{PDF}, f(\alpha)$ $\left[\mathrm{T}^{-1}\right]$, the expectant of $v_{\mathrm{d}}$ is found from

$$
\left\langle v_{\mathrm{d}}\right\rangle=\int_{0}^{\infty} v_{\mathrm{d}} f(\alpha) \mathrm{d} \alpha
$$

and

$$
\left\langle\frac{\mathrm{d} v_{\mathrm{d}}}{\mathrm{d} t}\right\rangle=\int_{0}^{\infty} \frac{\mathrm{d} v_{\mathrm{d}}}{\mathrm{d} t} f(\alpha) \mathrm{d} \alpha
$$

Here we consider the case that $f(\alpha)$ is a truncated power-law of the form

$$
f(\alpha)=\frac{(k-1) \alpha^{k-2}}{\alpha_{1}^{k-1}-\alpha_{0}^{k-1}}, \quad \alpha \in\left[\alpha_{0}, \alpha_{1}\right]
$$

where $k[-]$ is an exponent and $\alpha_{0}{ }^{\left[\mathrm{T}^{-1}\right]}$ and $\alpha_{1}{ }^{\left[\mathrm{T}^{-1}\right]}$ represent the minimum and maximum diffusion rates, respectively.

The multi-rate model applied in our study is based on the existence of a distribution of particle sizes, which implies a distribution of characteristic diffusion rates. The superposition of gas production rates originating from a set of particles of different size and shape gives rise to a different decay law at intermediate times whilst a cut-off time is determined by the largestsized particles. The reason for adopting a truncated power-law PDF for diffusion rates is based on the following observations: Firstly, there needs to be a truncation in the distribution due to the simple fact that there is a smallest and largest particle size within the mixture of particles, which implies a largest and smallest diffusion rate, respectively. Secondly, the observation of a power-law in the production rate at intermediate times indicates a power-law in the distribution of the diffusion rates. This becomes clear in the subsequent examination of Figs. 1 and 2 below.

Haggerty et al. (2000) previously derived analytical solutions for Eq. (9) with Eq. (10) for the special cases where $k=0,1$ and 2. They claim that solutions for non-integer $k$ values are only achievable using numerical methods. In fact, this is not true. We have derived such solutions by substituting Eqs. (3), (7) and (10) into Eqs. (8) and (9) and evaluating the resulting integrals. The resulting formulae take the form:

$$
\langle F\rangle \equiv \frac{\left\langle v_{\mathrm{d}}\right\rangle}{v_{\mathrm{d} 0}}=1-\frac{6}{\pi^{2}} \sum_{n=1}^{\infty} \frac{(k-1) \tau_{n}^{1-k}\left[\Gamma\left(k-1, \alpha_{0} \tau_{n}\right)-\Gamma\left(k-1, \alpha_{1} \tau_{n}\right)\right]}{n^{2}\left(\alpha_{1}^{k-1}-\alpha_{0}^{k-1}\right)}
$$

and 
(a) $k=0.75$

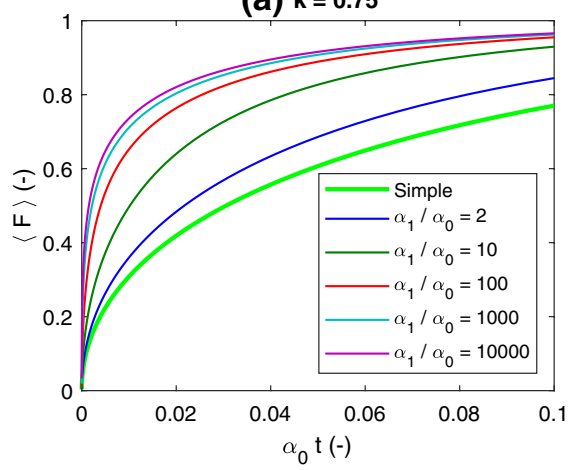

(c) $k=1.5$

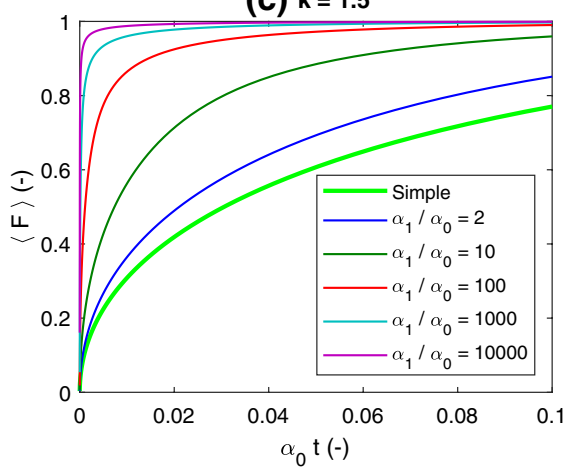

(e) $k=3$

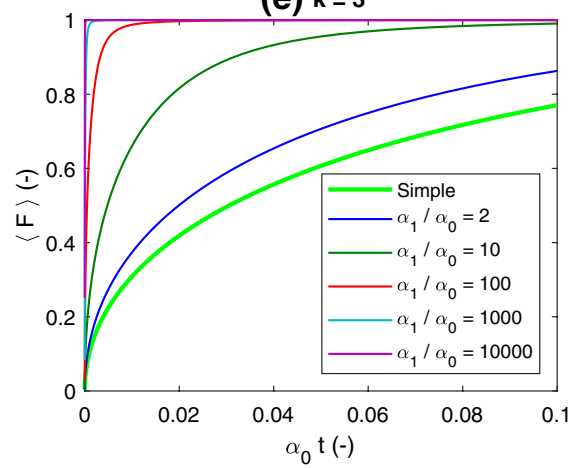

(b) $k=0.75$

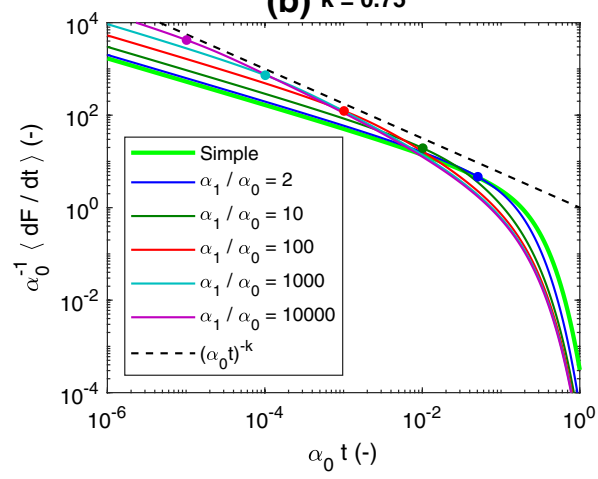

(d) $k=1.5$

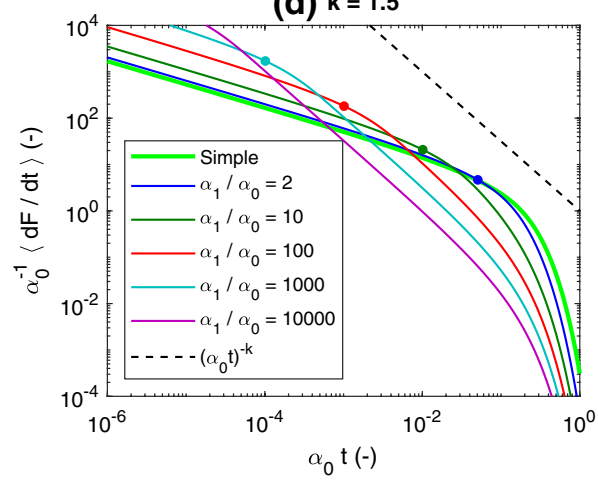

(f) $k=3$

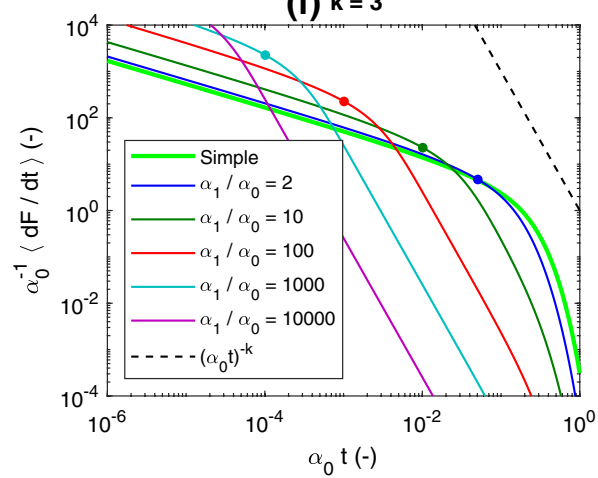

Fig. 1 Plots of $\langle F\rangle$ and $\langle\mathrm{d} F / \mathrm{d} t\rangle$, using the stochastic power-law model, for different values of $k$ (as indicated in the subtitles) and $\alpha_{1} / \alpha_{0}$ (as indicated in the legends). Results from the simple spherical diffusion model (simple) are shown for comparison as thick green lines. The stochastic power-law model log-log slopes are shown for comparison as black dashed lines. The coloured circular markers show where $t=\pi^{-2} \alpha_{1}^{-1}$, which marks the time at which the log-log slope, for the stochastic power-law model, is no longer equal to -0.5

$$
\left\langle\frac{\mathrm{d} F}{\mathrm{~d} t}\right\rangle \equiv \frac{1}{v_{\mathrm{d} 0}}\left\langle\frac{\mathrm{d} v_{\mathrm{d}}}{\mathrm{d} t}\right\rangle=6 \sum_{n=1}^{\infty} \frac{(k-1) \tau_{n}^{-k}\left[\Gamma\left(k, \alpha_{0} \tau_{n}\right)-\Gamma\left(k, \alpha_{1} \tau_{n}\right)\right]}{\alpha_{1}^{k-1}-\alpha_{0}^{k-1}}
$$

where $\Gamma(a, x)$ is the incomplete gamma function (Jameson 2016). 
(a)

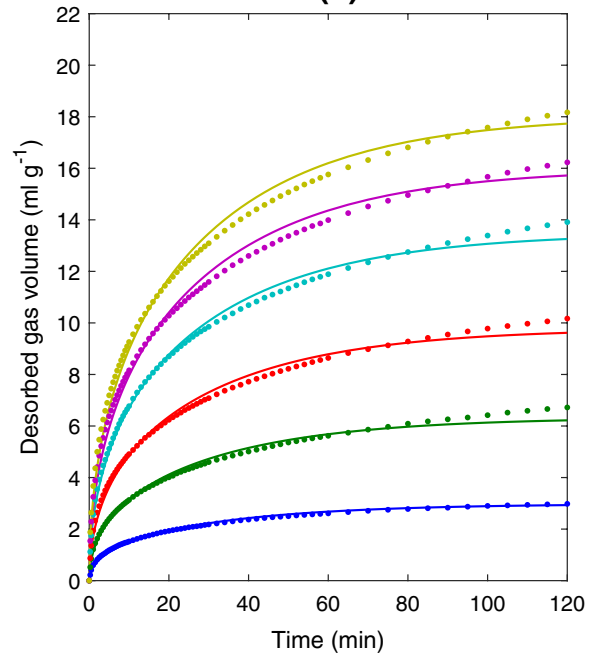

(c)

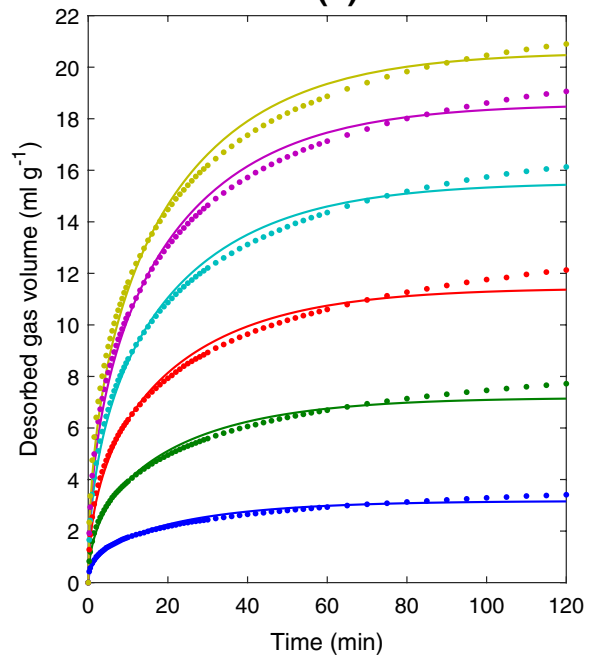

(b)

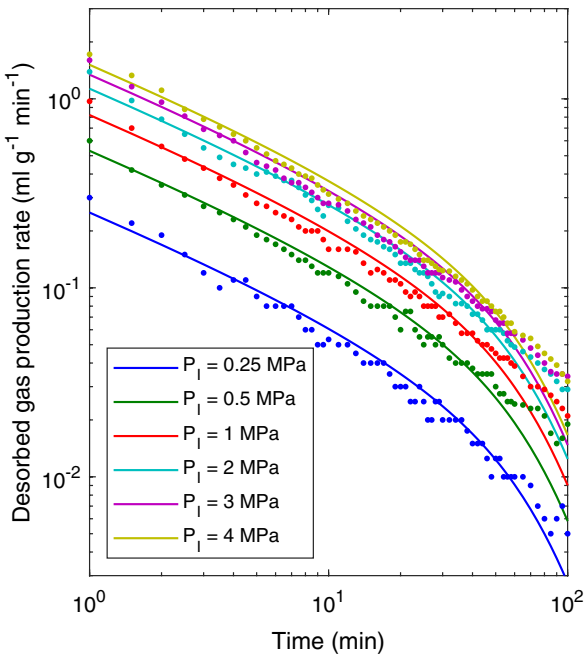

(d)

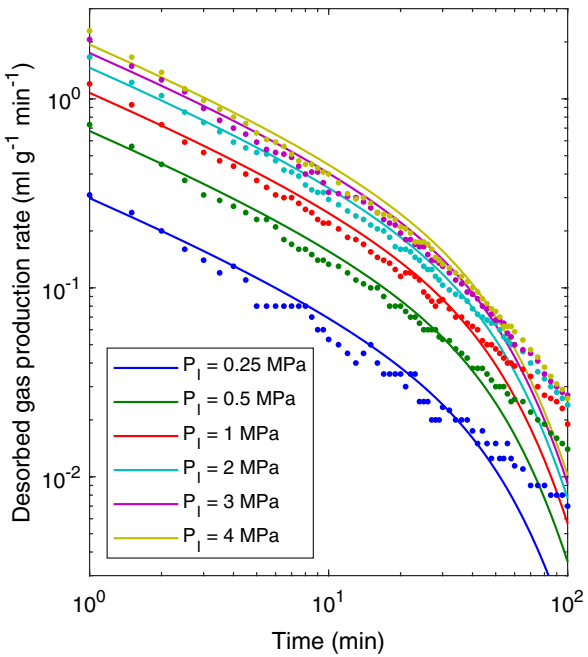

Fig. 2 Results from calibrating the simple spherical diffusion model to observed desorbed gas volume data. The solid lines and circular markers are results from the model and the observed data, respectively. a, b Results for sample 1. c, d Results for sample 2

Notably, Eq. (11) is problematic because $\Gamma(a, x)$ is difficult to evaluate for $a<0$. However, given the recursive relationship (Jameson 2016)

$$
\Gamma(a+1, x)=a \Gamma(a, x)+x^{a} e^{-x}
$$

it can be shown that 


$$
\langle F\rangle=1-\frac{6}{\pi^{2}} \sum_{n=1}^{\infty} \frac{\tau_{n}^{1-k}\left[\Gamma\left(k, \alpha_{0} \tau_{n}\right)-\Gamma\left(k, \alpha_{1} \tau_{n}\right)\right]+\alpha_{1}^{k-1} e^{-\alpha_{1} \tau_{n}}-\alpha_{0}^{k-1} e^{-\alpha_{0} \tau_{n}}}{n^{2}\left(\alpha_{1}^{k-1}-\alpha_{0}^{k-1}\right)}
$$

Fig. 1a, c and e shows example plots of $\langle F\rangle$ against normalized time, $\alpha_{0} t$, for a range of $k$ values and $\alpha_{1} / \alpha_{0}$ ratios. It can be seen that $\langle F\rangle$ equilibrates faster with increasing $\alpha_{1}$ and also increasing $k$. This is because increasing these parameters imply that the PDF for $\alpha$ is increasingly dominated by higher diffusion rates. Also of interest is that, regardless of the $k$ value, as $\alpha_{1} \rightarrow \alpha_{0}$, the stochastic power-law model converges on results from the simple spherical diffusion model (shown as a thick green line for comparison).

Fig. $1 \mathrm{~b}, \mathrm{~d}$ and $\mathrm{f}$ shows corresponding plots of $\alpha_{0}^{-1}\langle\mathrm{~d} F / \mathrm{d} t\rangle$ against normalized time, $\alpha_{0} t$, on $\log -\log$ axes. For $t<\pi^{-2} \alpha_{1}^{-1}$ (as indicated by the coloured circular markers), the $\log -\log$ slope is -0.5 , similar to the simple spherical diffusion model (again presented as thick green lines for comparison). For $\pi^{-2} \alpha_{1}^{-1}<t<\pi^{-2} \alpha_{0}^{-1}$, the log-log slope of the stochastic power-law model is $-k$ (compare with the plots of $\left(\alpha_{0} t\right)^{-k}$, shown as black dashed lines). For $t>\pi^{-2} \alpha_{0}^{-1}$, the gas production rate quickly drops off as this represents the time at which the system reaches diffusion equilibrium. The $\pi^{2}$ factor is a geometry parameter, characteristic of diffusion in spheres (Zimmerman et al. 1993).

The fact that $\langle\mathrm{d} F / \mathrm{d} t\rangle$ plots against $t$ on $\log -\log$ axes as a straight line with a slope of $-k$ for intermediate times is indicated by the presence of a $\tau_{n}^{-k}$ term in Eq. (12). However, because this happens at intermediate times as opposed to early or late times, it is not possible to derive a neat asymptotic result to demonstrate this point further.

\subsection{Joint Inversion Procedure}

In previous studies (e.g. Dong et al. 2017; Yue et al. 2017; Cheng-Wu et al. 2018), the simple spherical diffusion model, Eq. (3), has been fitted to gas desorption data with Langmuir isotherm parameters obtained a priori using additional steady state desorption data. Here, the gas desorption data of Dong et al. (2017) are revisited using Eq. (3) but with the diffusion rate, $\alpha$, and values of $v_{\mathrm{d} 0}$, for each initial pressure, $P_{\mathrm{I}}$, studied, treated as unknown parameters, obtained by joint inversion of gas desorption data for each $P_{\mathrm{I}}$ studied. This was achieved as follows:

MATLAB's optimization tool, FMINSEARCH, was used to select a value of $\alpha$, which in turn was used to determine values of $F$ from Eq. (3) for each time under consideration. A value of $v_{\mathrm{d} 0}$ was obtained by dividing the mean of the observed $v_{\mathrm{d}}$ values, for a given $P_{\mathrm{I}}$, by the mean of the $F$ values. This was repeated for each $P_{\mathrm{I}}$ value studied. A set of modelled $v_{\mathrm{d}}$ values was obtained, for each $P_{\mathrm{I}}$ value studied, by multiplying the $F$ values by each of $v_{\mathrm{d} 0}$ values. The root-mean-squared error (RMSE) between the modelled and observed $v_{\mathrm{d}}$ values was determined. FMINSEARCH then iteratively changed the value of $\alpha$ until the RMSE was minimized.

The above methodology was also applied using the stochastic power-law model, Eq. (14). However, in this case, FMINSEARCH was used to find optimal values for $\alpha_{0}$ and $\alpha_{1}$ with $k[-]$ being obtained a priori by inspection of the log-log slope of the observed $\mathrm{d} v_{\mathrm{d}} / \mathrm{d} t$ data.

FMINSEARCH is a local optimization tool, which is appropriate in this case because the number of free parameters is small and multiple minima in RMSE are not expected. FMINSEARCH requires specification of seed values for the unknown model parameters. These were obtained my manual, a priori, trial and error fitting of 
Fig. 3 Plot of estimated final desorbed gas volume, $v_{\mathrm{d} 0}$, against initial pressure of the desorption experiment, $P_{\mathrm{I}}$. The green line is the Langmuir isotherm from steady state adsorption data previously obtained by Dong et al. (2017). The other dashed lines and solid lines are estimates based on calibrating the simple spherical diffusion model (simple) and the stochastic power-law model (stochastic) to transient desorbed gas volume data, respectively

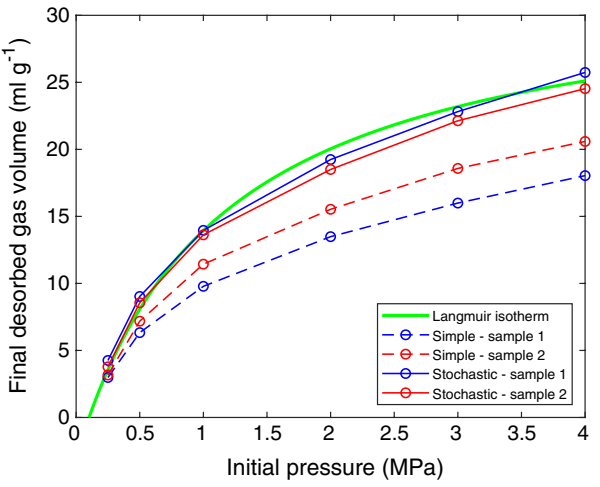

Table 1 Calibrated parameter values and associated RMSE using the simple spherical diffusion model (simple) and the stochastic power-law model (stochastic) for both sample 1 (1-3 mm) and sample $2(0.5-1$ $\mathrm{mm})$

\begin{tabular}{llllll}
\hline Model & $\alpha_{0}\left(\mathrm{~min}^{-1}\right)$ & $\alpha_{1}\left(\mathrm{~min}^{-1}\right)$ & $k(-)$ & $\mathrm{RMSE}\left(\mathrm{ml} \mathrm{g}^{-1}\right)$ & $D_{\mathrm{A}}\left(\mathrm{m}^{2} \mathrm{~s}^{-1}\right)$ \\
\hline Simple-sample 1 & 0.00302 & - & - & 0.243 & $5.03 \times 10^{-11}$ \\
Simple_sample 2 & 0.00391 & - & - & 0.289 & $9.16 \times 10^{-12}$ \\
Stochastic_-sample 1 & 0.000112 & 0.0431 & 0.70 & 0.0795 & $1.86 \times 10^{-12}$ \\
Stochastic_sample 2 & 0.000380 & 0.0371 & 0.70 & 0.102 & $8.91 \times 10^{-13}$ \\
\hline
\end{tabular}

$\alpha$ value for the simple spherical diffusion model is shown as $\alpha_{0}$ for convenience

the models to the observed data. For the simple spherical diffusion model, the seed value of $\alpha$ was taken to be $0.001 \mathrm{~min}^{-1}$. For the stochastic power-law mode, the seed values for $\alpha_{0}$ and $\alpha_{1}$ were taken to be $0.001 \mathrm{~min}^{-1}$ and $0.1 \mathrm{~min}^{-1}$, respectively.

\section{Results}

Comparisons of simulated and observed desorbed gas volume for samples 1 and 2, using the simple spherical diffusion model, are shown in Fig. 2a and c, respectively. Note that only one $\alpha$ value is assumed for each sample. However, separate $v_{\mathrm{d} 0}$ values are assumed for each $P_{\mathrm{I}}$ value considered (these are shown in Fig. 3). The model closely follows the desorbed gas volume data. The calibrated model parameters and estimated diffusion coefficients are presented in Table 1. The diffusion coefficients are calculated using Eq. (5) with $a$ set to the median value, which is $2 \mathrm{~mm}$ for Sample 1 and $0.75 \mathrm{~mm}$ for Sample 2 . These values are consistent with the range of diffusion coefficients previously determined by Dong et al. (2017, Table 2) using a transient diffusion coefficient. However, it is clear that the model underestimates desorption during late timand $d$ ). This has resultedes. The same is also true for desorbed gas production rate (see Fig. $2 \mathrm{~b}$ in the estimated values for $v_{\mathrm{d} 0}$ being significantly less than those predicted using the Langmuir isotherm of Dong et al. (2017) (see Fig. 3).

An important feature of the simple spherical diffusion model is that the log-log slope of a plot of desorbed gas production rate against time is -0.5 . In contrast, the observed data 
show a $\log -\log$ slope of around -0.7 (see Fig. 2b, d). Deviations from a $-0.5 \log -\log$ slope are indicative of multi-rate phenomena (Haggerty et al. 2000). Also of interest is that the observed gas production rates exhibit an exponential cut-off at large times, which can be represented by the minimum diffusion rate in our truncated power-law PDF described above.

An advantage of using the stochastic power-law model is that the log-log slope can be directly specified using the $k$ parameter. Based on the observed data, we chose to set $k=0.7$. The two additional calibration parameters, $\alpha_{0}$ and $\alpha_{1}$, were then obtained by

(a)

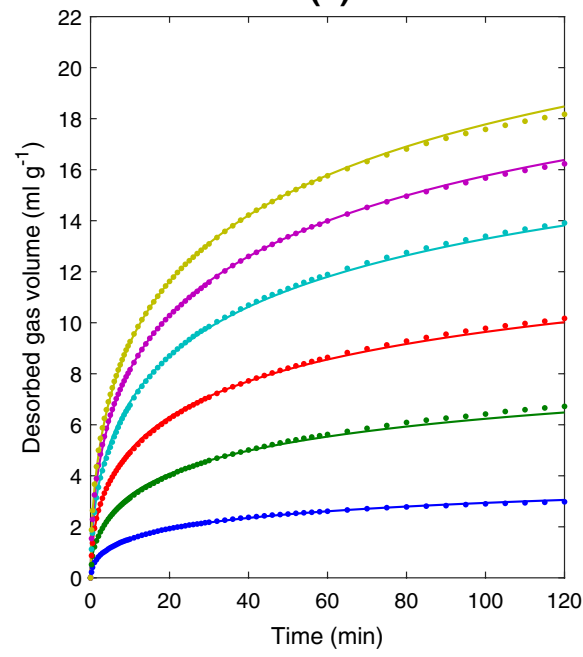

(c)

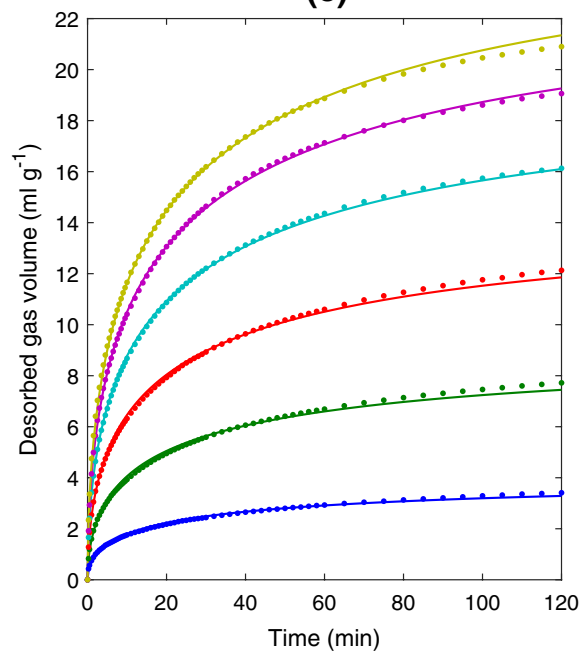

(b)

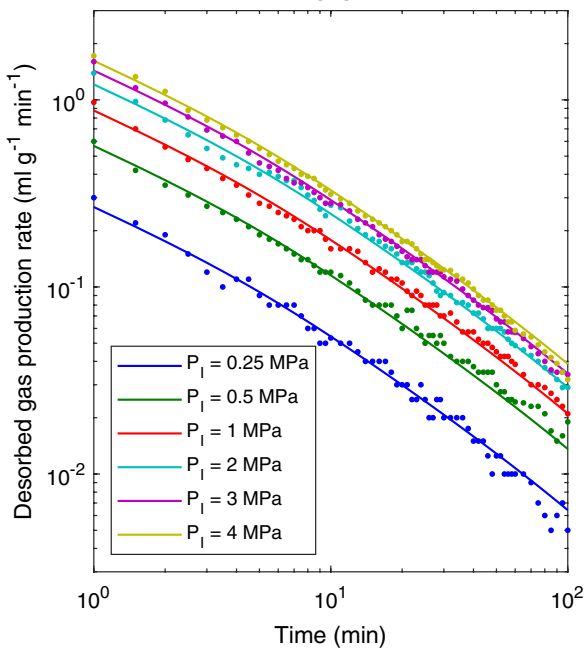

(d)

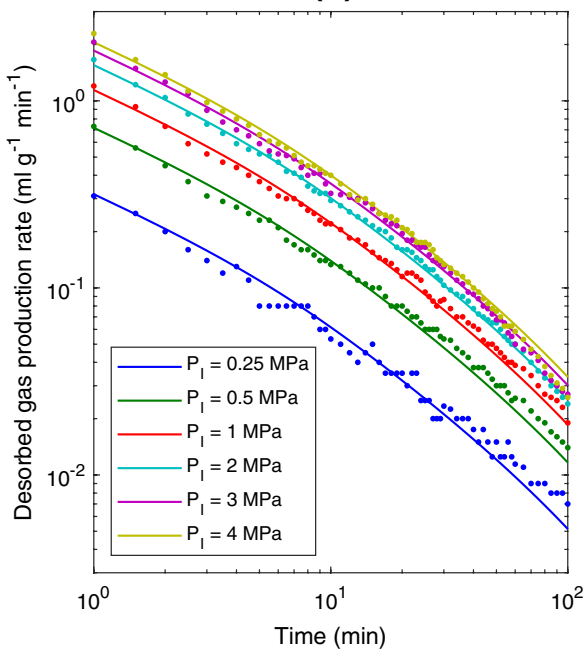

Fig. 4 Results from calibrating the stochastic power-law model to observed desorbed gas volume data. The solid lines and circular markers are results from the model and the observed data, respectively. a, b Results for sample 1. c, d Results for sample 2 
applying the calibration procedure described above. Comparisons of simulated and observed desorbed gas volume for samples 1 and 2, using the stochastic power-law model, are shown in Fig. 4a and c, respectively. Note that only one set of $\alpha_{0}$ and $\alpha_{1}$ values is assumed for each sample. However, separate $v_{\mathrm{d} 0}$ values are again determined for each $P_{\mathrm{I}}$ value considered. The model much more closely follows the observed desorption gas volume data (see Fig. 4a, c) as compared to the simple spherical diffusion model (compare RMSE values in Table 1). The same is also true for the desorbed gas production rate (compare Fig. $2 b$ and d with Fig. $4 b$ and d).

The calibrated model parameters, estimated diffusion coefficients and RMSE values are also presented in Table 1. The diffusion coefficients are calculated using Eq. (5) with $\alpha=\alpha_{0}$. These diffusion coefficients are much smaller than those estimated using the simple spherical diffusion model. This is because the stochastic power-law model also allows for the presence of much smaller particles, which have higher rate coefficients (as high as $\alpha_{1}$ ). It is also of note that the RMSE is significantly reduced when the stochastic powerlaw model is used instead of the simple spherical diffusion model (see Table 1). Furthermore, the estimated values for $v_{\mathrm{d} 0}$ are much closer to those predicted using the Langmuir isotherm of Dong et al. (2017) (see Fig. 3). Additionally, it is interesting to see that the observed data for all pressures plot as a single curve for each sample when the data are divided by the calibrated values of $v_{\mathrm{d} 0}$ (see Fig. 5).

\section{Conclusions}

Many previous studies claim that the apparent diffusion coefficient for surface diffusion of adsorbed gas in coal is a function of pressure and time. The objective of this study was to demonstrate that this diffusion coefficient can in fact be treated as a constant in both pressure and time. The demonstration involved revisiting gas desorption data previously obtained by Dong et al. (2017).

It was argued that the perceived pressure dependence of diffusion coefficient comes about because gas adsorption isotherms are obtained a priori to inversion of the diffusion coefficient. By acquiring the diffusion coefficient and the adsorption isotherm simultaneously from a joint inversion of gas desorption data from multiple initial pressures, it was hypothesized that the dependency of the diffusion coefficient on initial pressure would be removed.

It was further argued that the transient nature of the diffusion coefficient comes about due to missing physical processes in the mathematical models used for calibration. Typically, ground coal particles used for such experiments are treated as identical homogeneous spheres characterized by a single diffusion coefficient (i.e., the simple spherical diffusion model). Previous analyses of data from gas desorption experiments have focused on gas volume produced from the sample as a function of time plotted on linear axes. However, in this article, additional insight was gained by studying plots of gas production rate as function of time on $\log -\log$ axes. The simple spherical diffusion model manifests itself, at early times on such a plot, as a straight line with a slope of -0.5 . In contrast, the observed gas desorption data studied in this article exhibited a log-log slope of -0.7 . Deviations from a - 0.5 slope are indicative of multi-rate phenomena (Haggerty et al. 2000). Such phenomena can be explained in this context by the fact that individual coal particles comprise an agglomeration of multiple-sized sub-particles. 
(a)

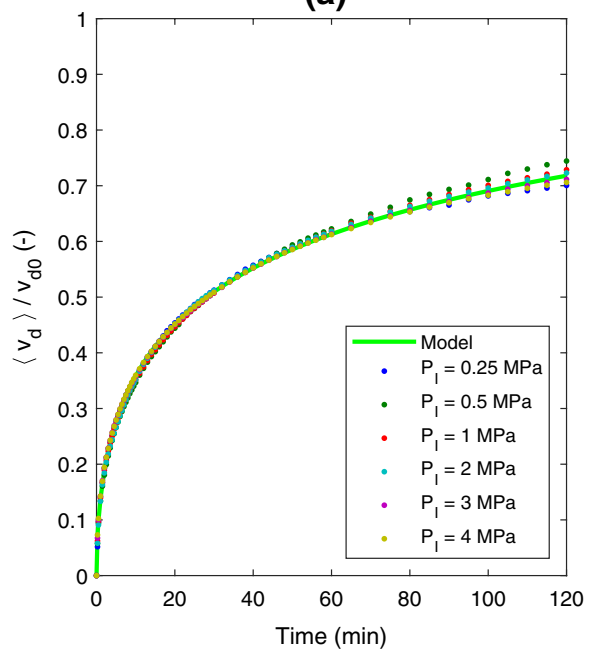

(c)

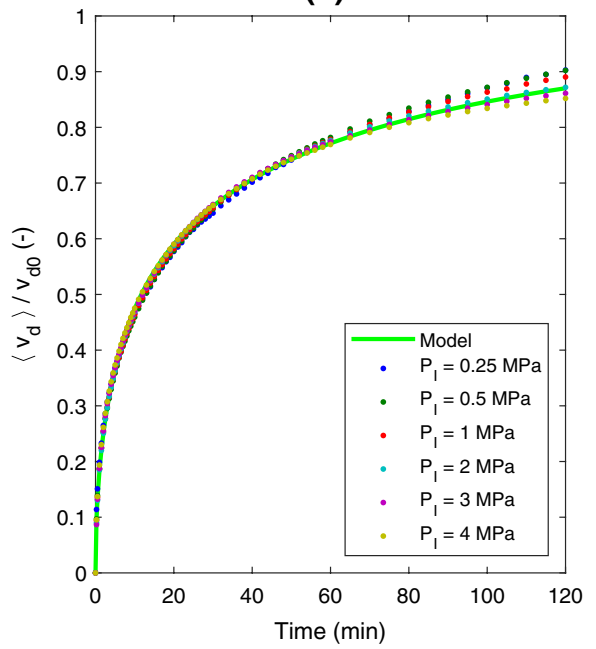

(b)

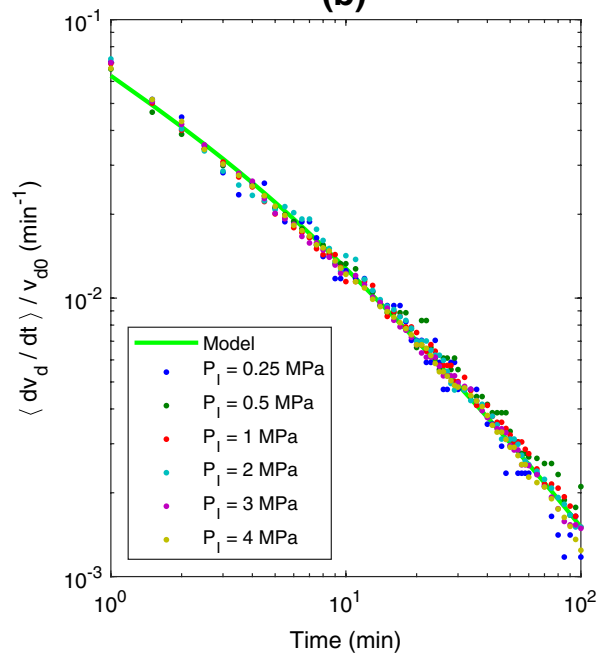

(d)

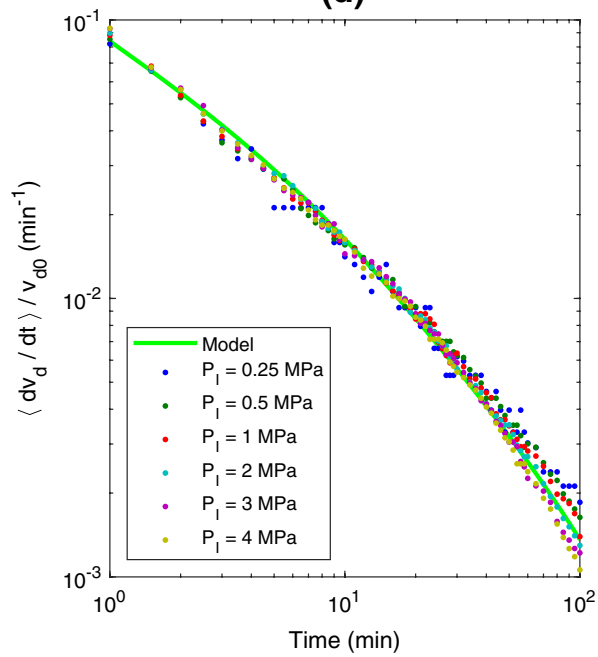

Fig. 5 Results from calibrating the stochastic power-law model to observed desorbed gas volume data rescaled by dividing by $v_{\mathrm{d} 0}$. The results from the model are shown as solid green lines. The observed data are shown as circular markers, with the different colours indicating the different initial pressures, as shown in the legends. a, b Results for sample 1. c, d Results for sample 2

The first joint inversion performed in this study employed the simple spherical diffusion model. A close correspondence between modelled and observed transient desorbed gas volume was obtained using a single static diffusion coefficient. However, closer inspection revealed that the model underestimates desorbed gas volume at late times. This in turn led to a significant underestimate of final desorbed gas volume as compared to that predicted by an a priori obtained Langmuir isotherm. 
Following on from this, a stochastic extension of the simple spherical diffusion model was derived, which assumes diffusion rates of spheres are described by a truncated powerlaw probability density function, referred to as the stochastic power-law model. The aforementioned $\log -\log$ slope from the stochastic power-law model was then set a priori to 0.7 , and an additional joint inversion was performed to obtain a new diffusion coefficient and adsorption isotherm. The correspondence between the model and observed transient desorbed gas volume data was significantly improved compared to when using the simple spherical diffusion model. Furthermore, the final desorbed gas volumes estimated using the stochastic power-law model were much closer to those predicted by the a priori obtained Langmuir isotherm.

The study relies on an assumption that MRP is due to individual coal particles comprising a conglomeration of smaller spherical particles with diffusion times characterized by a truncated power-law distribution. Furthermore, it is assumed that only surface diffusion occurs within the individual particles. Such an approach overlooks the potentially important roles of Knudsen diffusion and desorption of gas within the individual particles. Nevertheless, this limitation is common to most previous studies in the field. Furthermore, our proposed model captures experimental behaviour well using very few degrees of freedom.

An important conclusion from this study is that the apparent diffusion coefficient for surface diffusion of adsorbed gas in coal powder can be treated as a multi-rate process, which is constant in pressure and time. Furthermore, the study emphasizes the importance of studying plots of desorbed gas production rates against time on $\log -\log$ axes. It is also recommended to use the stochastic power-law model to determine surface diffusion coefficients for subsequent gas desorption studies.

Open Access This article is licensed under a Creative Commons Attribution 4.0 International License, which permits use, sharing, adaptation, distribution and reproduction in any medium or format, as long as you give appropriate credit to the original author(s) and the source, provide a link to the Creative Commons licence, and indicate if changes were made. The images or other third party material in this article are included in the article's Creative Commons licence, unless indicated otherwise in a credit line to the material. If material is not included in the article's Creative Commons licence and your intended use is not permitted by statutory regulation or exceeds the permitted use, you will need to obtain permission directly from the copyright holder. To view a copy of this licence, visit http://creativecommons.org/licenses/by/4.0/.

\section{References}

Cheng-Wu, L., Hong-Lai, X., Cheng, G., Wen-Biao, L.: Modeling and experiments for the time-dependent diffusion coefficient during methane desorption from coal. J. Geophys. Eng. 15(2), 315-329 (2018)

Clarkson, C.R., Bustin, R.M.: The effect of pore structure and gas pressure upon the transport properties of coal: a laboratory and modeling study. 2. Adsorption rate modeling. Fuel 78(11), 1345-1362 (1999)

Crank, J.: The Mathematics of Diffusion. Oxford University Press, Oxford (1979)

Dentz, M., Cortis, A., Scher, H., Berkowitz, B.: Time behavior of solute transport in heterogeneous media: transition from anomalous to normal transport. Adv. Water Resour. 27(2), 155-173 (2004)

Dong, J., Cheng, Y., Liu, Q., Zhang, H., Zhang, K., Hu, B.: Apparent and true diffusion coefficients of methane in coal and their relationships with methane desorption capacity. Energy Fuels 31(3), 2643-2651 (2017)

Fan, W., Jiang, X., Chen, S.: Parameter estimation for the fractional fractal diffusion model based on its numerical solution. Comput. Math. Appl. 71(2), 642-651 (2016)

Gouze, P., Melean, Y., Le Borgne, T., Dentz, M., Carrera, J.: Non-Fickian dispersion in porous media explained by heterogeneous microscale matrix diffusion. Water Resour. Res. 44, W11416 (2008)

Guo, H., Cheng, Y., Yuan, L., Wang, L., Zhou, H.: Unsteady-state diffusion of gas in coals and its relationship with coal pore structure. Energy Fuels 30(9), 7014-7024 (2016) 
Haggerty, R., McKenna, S.A., Meigs, L.C.: On the late-time behavior of tracer test breakthrough curves. Water Resour. Res. 36(12), 3467-3479 (2000)

Haggerty, R., Fleming, S.W., Meigs, L.C., McKenna, S.A.: Tracer tests in a fractured dolomite: 2. Analysis of mass transfer in single-well injection-withdrawal tests. Water Resour. Res. 37(5), 1129-1142 (2001)

Jameson, G.J.O.: The incomplete gamma functions. Math. Gaz. 100(548), 298-306 (2016)

Jiang, H., Cheng, Y., Yuan, L., An, F., Jin, K.: A fractal theory based fractional diffusion model used for the fast desorption process of methane in coal. Chaos Interdiscip. J. Nonlinear Sci. 23(3), 033111 (2013)

Kang, J., Zhou, F., Ye, G., Liu, Y.: An anomalous subdiffusion model with fractional derivatives for methane desorption in heterogeneous coal matrix. AIP Adv. 5(12), 127119 (2015)

Kang, J., Zhou, F., Xia, T., Ye, G.: Numerical modeling and experimental validation of anomalous time and space subdiffusion for gas transport in porous coal matrix. Int. J. Heat Mass Transf. 100, 747-757 (2016)

King, G.R., Ertekin, T., Schwerer, F.C.: Numerical simulation of the transient behavior of coal-seam degasification wells. SPE Form. Eval. 1(02), 165-183 (1986)

Koekemoer, A., Luckos, A.: Effect of material type and particle size distribution on pressure drop in packed beds of large particles: extending the Ergun equation. Fuel 158, 232-238 (2015)

Liu, J., Fokker, P.A., Spiers, C.J.: Coupling of swelling, internal stress evolution, and diffusion in coal matrix material during exposure to methane. J. Geophys. Res. Solid Earth 122(2), 844-865 (2017)

Liu, P., Qin, Y., Liu, S., Hao, Y.: Non-linear gas desorption and transport behavior in coal matrix: experiments and numerical modeling. Fuel 214, 1-13 (2018)

Liu, T., Lin, B.: Time-dependent dynamic diffusion processes in coal: model development and analysis. Int. J. Heat Mass Transf. 134, 1-9 (2019)

Meerschaert, M.M., Zhang, Y., Baeumer, B.: Tempered anomalous diffusion in heterogeneous systems. Geophys. Res. Lett. 35, L17403 (2008)

Miao, Y., Li, X., Zhou, Y., Wu, K., Chang, Y., Xiao, Z., et al.: A dynamic predictive permeability model in coal reservoirs: effects of shrinkage behavior caused by water desorption. J. Pet. Sci. Eng. 168, 533-541 (2018)

Smith, D.M., Williams, F.L.: Diffusion models for gas production from coals: application to methane content determination. Fuel 63(2), 251-255 (1984)

Wang, K., Zang, J., Feng, Y., Wu, Y.: Effects of moisture on diffusion kinetics in Chinese coals during methane desorption. J. Nat. Gas Sci. Eng. 21, 1005-1014 (2014)

Wang, G., Ren, T., Qi, Q., Lin, J., Liu, Q., Zhang, J.: Determining the diffusion coefficient of gas diffusion in coal: development of numerical solution. Fuel 196, 47-58 (2017)

Ye, Z., Chen, D., Wang, J.G.: Evaluation of the non-Darcy effect in coalbed methane production. Fuel 121, $1-10(2014)$

Yue, G., Wang, Z., Xie, C., Tang, X., Yuan, J.: Time-dependent methane diffusion behavior in coal: measurement and modeling. Transp. Porous Media 116(1), 319-333 (2017)

Zang, J., Wang, K.: A numerical model for simulating single-phase gas flow in anisotropic coal. J. Nat. Gas Sci. Eng. 28, 153-172 (2016)

Zang, J., Wang, K., Liu, A.: Phenomenological over-parameterization of the triple-fitting-parameter diffusion models in evaluation of gas diffusion in coal. Processes 7(4), 241 (2019)

Zhao, W., Cheng, Y., Jiang, H., Wang, H., Li, W.: Modeling and experiments for transient diffusion coefficients in the desorption of methane through coal powders. Int. J. Heat Mass Transf. 110, 845-854 (2017)

Zhao, W., Cheng, Y., Pan, Z., Wang, K., Liu, S.: Gas diffusion in coal particles: a review of mathematical models and their applications. Fuel 252, 77-100 (2019)

Zimmerman, R.W., Chen, G., Hadgu, T., Bodvarsson, G.S.: A numerical dual-porosity model with semianalytical treatment of fracture/matrix flow. Water Resour. Res. 29(7), 2127-2137 (1993)

Publisher's Note Springer Nature remains neutral with regard to jurisdictional claims in published maps and institutional affiliations. 